\title{
EDITORIAL
}

\section{Initiating therapeutic hypothermia during transport for encephalopathy: current state and future direction}

Journal of Perinatology (2013) 33, 169-170; doi:10.1038/jp.2012.168

Therapeutic hypothermia is the only treatment which has improved outcomes for late preterm and term infants with an encephalopathy of a hypoxic-ischemic origin in randomized clinical trials. Meta-analysis of outcomes assessed at a minimum age of 18 months for 1214 infants from seven trials indicated uniform benefit with a reduction in the risk of death or major neurodevelopmental disability (risk ratio (RR), 0.76; 95\% confidence interval $(\mathrm{Cl}): 0.69$ to 0.84$)$ and an increase in the rate of survival without neurological abnormality (RR 1.63, 95\% Cl: 1.36 to 1.95. ${ }^{1}$ ) It comes as no surprise that therapeutic hypothermia has been rapidly disseminated among neonatal care providers over recent years. This is not a therapy that can be offered by all hospitals. Infants with encephalopathy of a hypoxic-ischemic origin are typically critically ill with multi-system organ dysfunction and require care in neonatal intensive care units (NICU) with the necessary expertise, consultants and diagnostic services to manage the full spectrum of morbidities that may be triggered by hypoxia-ischemia. A similar infrastructure is needed to provide therapeutic hypothermia given the relative infrequency of this condition, the need to rapidly identify such infants and to initiate therapy within $6 \mathrm{~h}$ of birth as studied in clinical trials. For nursery services that do not provide therapeutic hypothermia, transport of infants to tertiary centers for this treatment is common and raises the issue of initiating hypothermia at the referring hospital or on transport. The latter practice allows provision of therapeutic hypothermia to infants who are geographically remote from centers providing this treatment, and can expedite initiation earlier in the limited therapeutic time window. Initiation of therapeutic hypothermia before or during transport represents an important issue for the dissemination of this therapy but is without clear data from completed clinical trials to guide this emerging practice.

The report by Akula et al. ${ }^{2}$ in this issue of the Journal of Perinatology provides important data regarding therapeutic hypothermia during neonatal transport. Two databases in California were used to examine transport practices and temperatures upon admission to centers providing therapeutic hypothermia and represents data from $>90 \%$ of newborns requiring NICU care in the state during 2010. Cooling on transport was common; $87 \%$ of out-born infants transferred for therapeutic hypothermia were cooled on transport with the majority initiated by the referring hospital and continued by the transport team. Modes of cooling at referring centers and on transport were either passive (removal of exogenous heat sources) or active (application of gel packs or ice to the body). Initiation of cooling by referring hospitals allowed a higher percent of infants to have therapy initiated before $6 \mathrm{~h}$ of age in contrast to cooling initiated either by the transport team or after admission to the tertiary center. However, only $44 \%$ of infants cooled on transport had core temperatures within the targeted range for therapeutic hypothermia upon tertiary center admission. Information is not available in the databases to indicate the criteria used to initiate therapeutic hypothermia.
Other single and multi-center reports document a similar spectrum of admission temperatures after hypothermia on transport. ${ }^{3,4}$ The data provided by Akula et al. ${ }^{2}$ extends prior publications by adding a larger data set extracted from a database with systematic collection of admission temperatures from multiple transport teams and NICUs. Initiation of therapeutic hypothermia at referring hospitals and on transport is a logical means to extend an effective therapy to more infants who are at risk for mortality or serious morbidity following hypoxia-ischemia. However, this report substantiates that implementation of hypothermia therapy during transport as currently practiced leads to wide scatter in achieved temperatures whereby infants with little temperature reduction are unlikely to benefit from any neuroprotection, and those with excessively low temperatures may be at risk for harm. The data emphasize that our ability to monitor and control temperature, a core vital sign, is limited on transport and has not kept abreast with other technologies used to monitor critically ill infants during transport.

There are a number of ways in which therapeutic hypothermia can be moved forward to provide a more effective treatment when used during the process of transport. An issue not dealt with in detail by Akula et al. ${ }^{2}$ is whether infants who undergo therapeutic hypothermia on transport are appropriate candidates for this therapy. All clinical trials to date have studied moderate or severe encephalopathy in the setting of clinical and physiological evidence supporting impairment of placental gas exchange and excluded infants with a mild encephalopathy. ${ }^{1}$ In the report by Akula et al., ${ }^{2} 16 \%$ of infants were judged to have a mild encephalopathy and $16 \%$ were without a diagnosis of encephalopathy. Although the latter may reflect, in part, limitations of the databases, there is scant published information on how and when infants are assessed for the presence of moderate or severe encephalopathy outside of clinical trials. Randomized studies such as the ICE and TOBY trials ${ }^{5,6}$ used trained retrieval teams to assess infants at the referring facility. Training transport team members is a viable strategy but even these providers need periodic re-education; even with updates transport personnel may have a minimal experience in newborn neurological examinations owing to infrequent assessments in the transport setting. In addition, transport teams may not arrive in time to initiate the therapy at $<6 \mathrm{~h}$ for some regions. Out-reach education for referring physicians can be used but may have limited impact for a diagnosis that occurs infrequently. Recognition of severe encephalopathy may be straight forward but distinguishing the effects of non-hypoxic-ischemic variables (for example, maternal analgesia/anesthesia) and more subtle examination abnormalities can be challenging. Videoconferencing and use of new communication technologies (smart phones, tablets and so on) can potentially share the neurological assessment by community providers with personnel at referral centers responsible for therapeutic hypothermia programs.

Assuming the appropriate infant is identified for initiation of therapeutic hypothermia before or during transport, pragmatic studies are needed to determine the optimal manner to achieve a targeted temperature reduction. Such studies can be performed in a randomized format or as a quality initiative to determine the feasibility of achieving goal temperatures within a pre-specified 
range over time. A central component of initiating and maintaining therapeutic hypothermia is continuous monitoring of core body temperature. If temperature monitoring is not compatible with transport incubators, free standing thermometers and temperature probes can be used. The challenges of frequent temperature monitoring by nurseries that do not care for high acuity patients should not be underestimated and may require a formatted sequence to guide temperature adjustments. The scatter in admission temperatures after cooling on transport as reviewed by Akula et $a l^{2}{ }^{2}$ indicate that passive and make shift active cooling are ineffective methods to achieve targeted temperature reductions. Their data support the studies of devices, which can regulate temperature either by manual or servo-regulated adjustments. Ideally such devices should have low and high temperature alarms as transport personnel may be occupied with other stabilization issues of critically sick infants.

Analysis of implementation of therapeutic hypothermia for neonatal encephalopathy of a hypoxic-ischemic origin in the United Kingdom suggests that it has been done in a manner consistent with the underlying clinical trials. ${ }^{7}$ The challenges to implement and disseminate therapeutic hypothermia may differ in larger countries with diverse geography, referral patterns and health care systems. Therapeutic hypothermia initiated before or during transport has become a prevalent practice in the United States based on the data from California. What are needed at present are evidence-based guidelines for the optimal means to conduct hypothermia on transport after identification of infants appropriate for the therapy. Pragmatic studies of therapeutic hypothermia during transport combined with systematic data collection will provide more rigorous evidence to create clinical pathways for this intervention. The use of therapeutic hypothermia during transport, as currently practiced, although well intended, frequently does not achieve its purpose.

\section{CONFLICT OF INTEREST}

The author declares no conflict of interest.

AR Laptook

Department of Pediatrics, The Warren Alpert Medical School of Brown University, Medical Director, Neonatal Intensive Care Unit, Women and Infants Hospital of Rhode Island, Providence, RI, USA

E-mail: alaptook@wihri.org

\section{REFERENCES}

1 Tagin MA, Woolcott CG, Vincer MJ, Whyte RK, Stinson DA. Hypothermia for neonatal hypoxic ischemic encephalopathy: an updated systematic review and metaanalysis. Arch Pediatr Adolesc Med 2012; 166(6): 558-566.

2 Akula VP, Gould JB, Davis AS, Hackel A, Oehlert J, VanMeurs KP. Therapeutic hypothermia during neonatal transport: Data from the California Perinatal Quality Care Collaborative (CPQCC) and California Perinatal Transport System (CPeTS) for 2010. J Perinatol 2012 (e-pub ahead of print 6 December 2012).

3 Fairchild K, Sokora D, Scott J, Zanelli S. Therapeutic hypothermia on neonatal transport: 4-year experience in a single NICU. J Perinatol 2010; 30(5): 324-329.

4 Kendall GS, Kapetanakis A, Ratnavel N, Azzopardi D, Robertson NJ. Passive cooling for initiation of therapeutic hypothermia in neonatal encephalopathy. Arch Dis Child Fetal Neonatal Ed 2010; 95(6): F408-F412.

5 Jacobs SE, Morley CJ, Inder TE, Stewart MJ, Smith KR, McNamara PJ et al. Wholebody hypothermia for term and near-term newborns with hypoxic-ischemic encephalopathy: a randomized controlled trial. Arch Pediatr Adolesc Med 2011; 165(8): 692-700.

6 Azzopardi DV, Strohm B, Edwards AD, Dyet L, Halliday HL, Juszczak E et al. Moderate hypothermia to treat perinatal asphyxial encephalopathy. N Engl J Med 2009; 361(14): 1349-1358.

7 Azzopardi D, Strohm B, Linsell L, Hobson A, Juszczak E, Kurinczuk JJ et al. Implementation and conduct of therapeutic hypothermia for perinatal asphyxial encephalopathy in the UK--analysis of national data. PLoS One 2012; 7(6): e38504. 